\section{Descrição do consumo de psicofármacos na
atenção primária à saúde de Ribeirão Preto, \\ Descrição do consumo de psicofármacos na
atenção primária à saúde de Ribeirão Preto, São Paulo, Brasil}

\author{
Consumption of psychotropic medications in \\ primary healthcare in Ribeirão Preto, São Paulo \\ State, Brazil
}

\section{Descripción del consumo de psicofármacos en la atención primaria en salud de Ribeirão Preto, estado de São Paulo, Brasil}

\author{
Júlia Raso Ferreira de Oliveira 1 \\ Fabiana Rossi Varallo 1 \\ Marcela Jirón ${ }^{2}$ \\ Iahel Manon de Lima Ferreira 1 \\ Manuela Roque Siani-Morello 1 \\ Vinícius Detoni Lopes 1 \\ Leonardo Régis Leira Pereira 1
}

\section{Resumo}

O consumo de psicofármacos é considerado um problema de saúde pública devido ao potencial de dependência e ocorrência de eventos adversos. Nesse contexto, o presente estudo teve como objetivo caracterizar o consumo de psicofármacos dispensados em unidades básicas de saúde de Ribeirão Preto, São Paulo, Brasil. Conduziu-se um estudo ecológico, com consulta à base de dados Hygia de 2008 a 2012. Foram extraídas as variáveis: psicofármaco dispensado, quantidade dispensada no ano, sexo e faixa etária dos pacientes. Para cada psicofármaco foi calculada a dose diária definida por 1.000 habitantes/ dia (DDD/1.000PD), a dose diária definida por 1.000 habitantes/dia considerando-se $75 \%$ da população (DDD75\%/1.000PD) que retiraram medicamento pelo Sistema Único de Saúde (SUS) e a dose diária prescrita (DDP). Comparou-se a taxa de crescimento populacional com a de crescimento do consumo dos medicamentos. Foram identificados 1.577 .241 pacientes que retiraram medicamentos no período avaliado, dos quais 287.373 (18,2\%) utilizaram pelo menos um sujeito a controle especial. Houve aumento do consumo total dos psicofármacos (DDD/1.000PD), porém, após a comparação com a taxa de crescimento populacional, apenas a da sertralina $(p=0,021)$, risperidona $(p=$ $0,034)$ e do clonazepam ( $p=0,043)$ foram superiores. As DDP de sete fármacos estavam maiores que a DDD da Organização Mundial da Saúde (OMS). As discrepâncias entre DDD e DDP podem ser úteis como estratégia para triar pacientes elegíveis ao cuidado farmacêutico, pois podem contribuir na prevenção de morbimortalidade relacionada ao uso de medicamentos.

Psicotrópicos; Farmacoepidemiologia; Uso de Medicamentos; Sistema Único de Saúde

\author{
Correspondência \\ V. D. Lopes \\ Departamento de Ciências Farmacêuticas, Faculdade de \\ Ciências Farmacêuticas de Ribeirão Preto, Universidade de \\ São Paulo. \\ Av. do Café s/n, Ribeirão Preto, SP 14040-900, Brasil. \\ vinicius.detoni91@gmail.com \\ 1 Faculdade de Ciências Farmacêuticas de Ribeirão Preto, \\ Universidade de São Paulo, Ribeirão Preto, Brasil. \\ 2 Facultad de Ciencias Quimicas y Farmacéuticas, Universidad \\ de Chile, Santiago, Chile.
}




\section{Introdução}

No Brasil, os indivíduos que apresentam transtornos mentais recebem atendimento na Rede de Atenção Psicossocial (RAPS) que é formada por diversos pontos de atenção (serviços), dentre os quais se destacam os Centros de Atenção Psicossocial (CAPS), que compreendem o principal dispositivo de referência e tratamento para pessoas com transtornos mentais severos e persistentes.

Os CAPS que dispõem de farmácia realizam a dispensação de psicofármacos para usuários do Sistema Único de Saúde (SUS) a fim de facilitar o acesso ao medicamento, além disso, os profissionais que atuam no CAPS realizam intervenções para a reinserção do indivíduo no contexto social e familiar.

Os psicofármacos são substâncias químicas, naturais ou sintéticas, que quando introduzidas no organismo podem modificar de várias maneiras o comportamento mental, excitando, deprimindo ou provocando perturbações ${ }^{1}$. São indicados para o tratamento de diversas enfermidades e podem causar dependência física e/ou psíquica, além de contribuírem para a ocorrência de eventos adversos aos usuários.

Nos últimos anos, observa-se aumento no consumo desses medicamentos, o que pôde ser evidenciado na Pesquisa Nacional sobre Acesso, Utilização e Promoção do Uso Racional de Medicamentos no Brasil (PNAUM), que demonstrou que dentre os 20 subgrupos farmacológicos mais utilizados pelos usuários da atenção primária estão os antidepressivos (fluoxetina), antiepiléticos e ansiolíticos (clonazepam), ficando atrás apenas dos fármacos das classes de anti-inflamatórios não esteroidais, anti-hipertensivos e antidiabéticos 2 .

Esse consumo elevado pode ser explicado devido ao crescente número de diagnósticos de transtornos psiquiátricos na população, ao ingresso de novos psicofármacos no mercado farmacêutico e às novas indicações terapêuticas para psicotrópicos já existentes ${ }^{3}$. Contudo, também há relato na literatura sobre prescrição exacerbada desses medicamentos para determinados diagnósticos, tais como: depressão, transtorno bipolar e hiperatividade 4 . Nesses casos, é possível propor estratégias para promover mudanças de comportamento para o uso apropriado de medicamentos.

Um estudo multicêntrico evidenciou diferenças na prática de prescrição de medicamentos para doenças mentais em centros de atenção primária de países europeus, africanos e latino-americanos (inclusive o Brasil), principalmente quando se considerou o uso de polifarmácia, medicamentos tranquilizantes (ansiolíticos e hipnóticos) e antidepressivos 5 .

Estimativa demonstra que um em cada 10 brasileiros usa benzodiazepínicos durante a vida como tranquilizante ou indutor de sono. Os fatores de risco sociodemográficos identificados são sexo feminino ( $\mathrm{p}=0,001)$, os grupos etários entre 40-59 anos $(\mathrm{p}=0,002)$ e idosos $(\mathrm{p}=0,009)$ 6. Os benzodiazepínicos são um dos medicamentos mais utilizados no mundo e o seu uso cresce, principalmente entre as mulheres adultas e idosas 7 . Entretanto, há relatos de que o uso crônico aumenta o risco de desenvolvimento de demência, principalmente em idosos 8 .

Um estudo indica que a população brasileira tem a maior prevalência de depressão e ansiedade da América Latina, com taxas de 5,8\% e 9,3\%, respectivamente 9 . Por outro lado, os transtornos psiquiátricos, especialmente depressão, têm sido sobrediagnosticados em uma escala considerável, levando a uma lista de consequências adversas significativas que afetam principalmente os grupos de indivíduos mais vulneráveis 10 .

Esses achados sugerem que a utilização de medicamentos no Brasil, inclusive a dos psicofármacos, tem sido exacerbada e indiscriminada 6 . Observa-se indicação abusiva de medicamentos para sofrimentos psíquicos que, muitas vezes, estão relacionados a problemas sociais e econômicos. Sendo assim, o que se constata nos serviços de saúde mental é uma terapêutica ineficiente associada ao uso dos psicotrópicos, com frágil comunicação entre profissionais e usuários e pouco uso de tecnologias leves e leve-duras 11.

Assim, considerando-se os riscos associados ao uso de psicofármacos e a possibilidade de ocorrência de iatrogenias, as quais podem ocasionar eventos graves aos usuários, é necessário realizar estudos sobre a utilização de medicamentos, com o intuito de gerar hipóteses associadas ao aumento do consumo e, assim, contribuir para o seu uso seguro e apropriado.

Nesse contexto, os objetivos deste trabalho foram: descrever o consumo de psicofármacos dispensados nas unidades básicas de saúde do SUS do Município de Ribeirão Preto, São Paulo, Brasil, comparar se os valores médios das doses diárias prescritas (DDP) são semelhantes aos valores das 
doses diárias definidas (DDD) da Organização Mundial da Saúde (OMS) e correlacionar o uso de medicamentos com a taxa de crescimento populacional.

\section{Métodos}

\section{Tipo e local do estudo}

Foi conduzido um estudo epidemiológico, do tipo descritivo e ecológico sobre o consumo de psicofármacos dispensados nas unidades básicas de saúde do Município de Ribeirão Preto. A série temporal compreendeu o período de 2008 a 2012.

\section{Participantes}

Todos os pacientes que retiraram pelo menos um medicamento, em quaisquer unidades básicas de saúde do Município de Ribeirão Preto, foram considerados elegíveis. Contemplaram os critérios de inclusão aqueles que tiveram registro de dispensação de pelo menos um medicamento da linha de cuidado em saúde mental, ou seja, sujeitos a controle especial ou psicofármacos.

Apesar do registro de dispensação não indicar, necessariamente, o consumo real dos psicofármacos pela população, assumiu-se no presente estudo que os registros de retirada de medicamentos representavam uma estimativa dos dados de consumo dos participantes da pesquisa, pois o desenho epidemiológico adotado não permite afirmar se o paciente realmente usou o medicamento de acordo com a prescrição médica.

\section{Variáveis}

Os pacientes recrutados tiveram seus registros de dispensação analisados para a extração de variáveis demográficas (sexo e idade) e farmacológicas (psicofármaco dispensado, quantidade retirada e dose). Foram também coletados dados populacionais do município (número de habitantes nos anos de 2008, 2009, 2010, 2011 e 2012).

\section{Fontes de dados e mensuração}

Os dados demográficos e farmacológicos foram extraídos por meio de consulta retrospectiva aos registros de dispensação armazenados no banco de dados online - Hygia (http://www.hygia.com.br/). Nessa plataforma são armazenadas informações sobre os registros de dispensação de medicamentos e vacinas, bem como consultas médicas, exames laboratoriais, entre outros, para cada paciente atendido pelo SUS no Município de Ribeirão Preto.

Para a consulta do número total de habitantes em Ribeirão Preto, por ano, foram pesquisados os dados do Instituto Brasileiro de Geografia e Estatística 12.

Os medicamentos sujeitos a controle especial dispensados e registrados no banco de dados Hygia foram estratificados segundo a classificação Anatômico Terapêutico e Química (ATC). De acordo com a idade, os pacientes foram categorizados em: pediátricos ( 0 a 19 anos), adultos (20 a 59 anos) e idosos (maior ou igual a 60 anos).

O consumo do medicamento e a média de medicamento anual retirada por cada usuário foram estimados em miligramas/dia. As métricas para a estimativa de utilização empregadas no presente estudo foram:

a) Dose diária definida por 1.000 habitantes/dia (DDD/1.000PD). Para tanto, foi realizado o seguinte cálculo:

$$
\frac{\mathrm{DDD}}{1.000 \mathrm{PD}}=\left(\frac{\text { miligramas anual do psicofármaco }}{\mathrm{DDD} \times 365 \times \text { população anual }}\right) \times 1.000
$$


em que: miligrama anual = total de miligramas dispensadas do psicofármaco no ano; DDD = dose diária definida estabelecida pela OMS.

b) Dose diária definida por 1.000 habitantes/dia corrigida para tamanho da população que utiliza o SUS, ou seja, 75\% (DDD75\%/1.000PD). Para tanto, foi realizado o seguinte cálculo:

$$
\frac{\mathrm{DDD}}{1.000 \mathrm{PD}}=\left(\frac{\text { miligramas anual do psicofármaco }}{\mathrm{DDD} \times 365 \times 75 \% \text { da população anual }}\right) \times 1.000
$$

c) Dose diária prescrita (DDP):

$$
\mathrm{DDP}=\frac{\text { média anual das doses prescritas do psicofármaco em miligramas }}{\text { número de dias que o paciente utilizou o psicofármaco }}
$$

Para correlacionar o crescimento populacional com o consumo de psicofármacos foram calculadas a taxa de crescimento da população do município e a taxa do crescimento do número de pacientes em uso de psicofármacos. Para tanto, os seguintes raciocínios foram utilizados:

a) Taxa de crescimento populacional (TC):

$$
\mathrm{TC}=\frac{\text { número total de habitantes em } 2009-\text { número total de habitantes em } 2008}{\text { número total de habitantes em } 2008}
$$

b) Taxa de crescimento (TC) do número de pacientes em uso de psicofármacos (PF):

$$
\mathrm{TCPF}=\frac{\text { número total de pacientes em uso de PF em } 2009-\text { número total de pacientes em uso de PF em } 2008}{\text { número total de pacientes em uso de PF em } 2008}
$$

\section{Análise estatística}

Para avaliar se houve diferença estatística na DDP de psicofármacos em relação ao sexo foi aplicado o teste estatístico t de Student não pareado, considerando a métrica de consumo como variável dependente.

Para avaliar a correlação entre o aumento da idade e o consumo de psicofármacos foi realizada regressão linear simples, considerando a dose média do medicamento como a variável dependente e a idade como variável independente.

Com o intuito de verificar se a taxa de crescimento populacional superou ou não a taxa de crescimento de pacientes em uso de medicamentos sujeitos a controle especial, foi aplicado o teste estatístico não paramétrico de Mann-Whitney para amostras não pareadas.

\section{Aprovação por Comitê de Ética em Pesquisa}

O presente trabalho foi aprovado pelo Comitê de Ética em Pesquisa da Universidade de São Paulo (USP), sob o número do processo 228/2012. Estudo realizado sem conflitos de interesse.

\section{Resultados}

Durante o período de coleta de dados, 1.577.241 pacientes retiraram medicamentos no componente básico da assistência farmacêutica (CBAF) em Ribeirão Preto, dos quais 287.373 (18,2\%) utilizaram pelo menos um medicamento sujeito a controle especial. Desses, a maioria era do sexo feminino [203.094/287.373 (70,7\%)] e adultos [185.929/287.373 (64,7\%)] (Tabela 1).

Foram identificados 24 fármacos sujeitos a controle especial padronizados na relação municipal, das seguintes classes terapêuticas: (i) antidepressivos (amitriptilina, bupropiona, clomipramina, fluoxetina, imipramina, nortriptilina e sertralina); (ii) antiepilépticos (ácido valproico, carbamazepina, fenitoína e fenobarbital); (iii) ansiolíticos (diazepam e clonazepam); (iv) hipnótico-sedativo (nitrazepam); (v) antipsicóticos (carbonato de lítio, clorpromazina, haloperidol, levomepromazina, peri- 
Tabela 1

Frequência dos pacientes que retiraram medicamentos sujeitos a controle especial do componente básico da assistência farmacêutica. Município de Ribeirão Preto, São Paulo, Brasil.

\begin{tabular}{|c|c|c|c|c|c|c|c|c|c|c|c|c|}
\hline \multirow[t]{3}{*}{ Ano } & \multirow[t]{3}{*}{ Habitantes } & \multirow{3}{*}{$\begin{array}{l}\text { Usuários que } \\
\text { retiraram } \\
\text { medicamentos }\end{array}$} & \multirow{2}{*}{\multicolumn{2}{|c|}{$\begin{array}{l}\text { Pacientes que } \\
\text { retiraram } \\
\text { medicamentos } \\
\text { psicotrópicos }\end{array}$}} & \multicolumn{2}{|c|}{ Sexo feminino } & \multicolumn{6}{|c|}{ Faixa etária (anos) } \\
\hline & & & & & & & \multicolumn{2}{|c|}{$0-19$} & \multicolumn{2}{|c|}{$20-59$} & \multicolumn{2}{|c|}{$\geq 60$} \\
\hline & & & $\mathbf{n}$ & $\%$ & $\mathbf{n}$ & $\%$ & $\mathbf{n}$ & $\%$ * & $\mathbf{n}$ & $\%$ & $\mathbf{n}$ & $\%$ \\
\hline 2008 & 558.136 & 276.644 & 48.778 & 17,6 & 34.620 & 71,0 & 2.813 & 5,77 & 32.509 & 66,6 & 13.456 & 27,6 \\
\hline 2009 & 563.107 & 300.990 & 53.475 & 17,8 & 36.954 & 69,1 & 4.256 & 7,96 & 35.218 & 65,9 & 14.001 & 26,2 \\
\hline 2010 & 604.682 & 317.933 & 57.899 & 18,2 & 40.920 & 70,7 & 3.336 & 5,76 & 36.883 & 63,7 & 17.675 & 30,5 \\
\hline 2011 & 612.339 & 334.653 & 62.392 & 18,6 & 44.514 & 71,3 & 3.220 & 5,16 & 40.048 & 64,2 & 19.112 & 30,6 \\
\hline 2012 & 619.746 & 347.021 & 64.829 & 18,7 & 46.086 & 71,1 & 3.210 & 4,95 & 41.271 & 63,7 & 20.348 & 31,4 \\
\hline Total & 2.958 .010 & 1.577 .241 & 287.373 & 18,2 & 203.094 & 70,7 & 16.835 & 5,86 & 185.929 & 64,7 & 84.592 & 29,4 \\
\hline
\end{tabular}

Fonte: dados coletados do banco de dados online Hygia (http://www.hygia.com.br/).

* Em relação aos pacientes que retiraram medicamentos psicotrópicos.

ciazina, risperidona, tioridazina); (vi) anticolinérgicos (biperideno); (vi) dopaminérgicos (levodopa e associações); e (viii) antagonista opioide puro (naltrexona).

Quando se comparou o consumo total dos psicofármacos, foi verificado aumento na DDD/1000PD. Com relação à DDP, sete fármacos (carbonato de lítio, fenobarbital, fluoxetina, haloperidol, naltrexona, nitrazepam e sertralina) apresentaram o valor médio do consumo em DDP superior à DDD sugerida pela OMS (Tabela 2).

Evidência de aumento significativo do consumo, comparando-se a DDP do ano de 2012 com a do período de 2008-2011, foi identificada para amitriptilina, bupropiona, clonazepam, fenitoína, fenobarbital, fluoxetina, haloperidol e levodopa (Tabela 3). Evidenciou-se redução para clorpromazina, levomepromazina, naltrexona e tioridazina (Tabela 3).

Foi possível observar aumento do consumo, calculado pela DDD/1000PD e pela DDD75\%/1000PD para ácido valproico, carbonato de lítio, clomipramina, clonazepam, fenitoína, haloperidol, imipramina, naltrexona, nortriptilina, risperidona e sertralina. (Tabela 4). Em contrapartida, verificou-se a diminuição do consumo da fluoxetina (Tabela 4).

As mulheres consumiram dose média mais elevada do diazepam $(\mathrm{p}<0,001)$ e periciazina $(\mathrm{p}<$ 0,001); já os homens do ácido valproico ( $<<0,001)$, amitriptilina $(\mathrm{p}<0,001)$; carbamazepina $(\mathrm{p}<$ $0,001)$; carbonato de lítio ( $<<0,001)$; clonazepam ( $\mathrm{p}<0,001)$; clorpromazina $(\mathrm{p}<0,001)$; fenitoína ( $\mathrm{p}$ $<0,001)$; levodopa $(\mathrm{p}<0,001)$, risperidona $(\mathrm{p}<0,001)$ e tioridazina $(\mathrm{p}<0,001)$.

Verificou-se que com o aumento da idade ocorreu o aumento da dose do ácido valproico ( $\mathrm{p}<$ 0,001); diazepam ( $<<0,001)$; fenobarbital $(\mathrm{p}<0,001)$; imipramina $(\mathrm{p}<0,001)$; periciazina $(\mathrm{p}<0,001)$ e tioridazina ( $<$ 0,001). Respectivamente, o aumento de um ano na idade aumentou em 1,969mg/dia; em 0,084mg/dia; em 0,596mg/dia; em 0,051mg/dia; em 0,421 mg/dia e em 1,287mg/dia nas doses.

Por outro lado, o avanço da idade diminuiu a dose média do biperideno ( $p=0,029)$; carbamazepina ( $\mathrm{p}<0,001)$; carbonato de lítio $(\mathrm{p}=0,005)$; clonazepam $(\mathrm{p}<0,001)$; clorpromazina $(\mathrm{p}<0,001)$; fenitoína ( $\mathrm{p}<0,001)$; levomepromazina $(\mathrm{p}<0,001)$; risperidona $(\mathrm{p}<0,001)$ e sertralina $(\mathrm{p}<0,001)$. Respectivamente, o aumento em um ano na idade reduziu a dose em 0,073mg/dia; em 0,732mg/dia; em 1,794mg/dia; em 0,005mg/dia; em 0,305mg/dia; em 0,268mg/dia; em 0,296mg/dia; em 0,011mg/ dia; em 0,162mg/dia.

Apesar de ter havido aumento no consumo geral (DDD/1.000PD) nos cinco anos estudados, foi possível verificar que a taxa de crescimento no uso de sertralina, risperidona e clonazepam superou a taxa de crescimento populacional. Para os demais psicofármacos não houve diferença estatística (Tabela 5). 
Tabela 2

Comparação do consumo de medicamentos sujeitos a controle especial padronizados no componente básico da assistência farmacêutica do Município de Ribeirão Preto, São Paulo, Brasil.

\begin{tabular}{|c|c|c|c|c|c|c|c|}
\hline \multirow[t]{2}{*}{ Fármaco } & \multirow[t]{2}{*}{ DDD } & \multicolumn{6}{|c|}{ DDP } \\
\hline & & 2008 & 2009 & 2010 & 2011 & 2012 & Média \\
\hline Ácido valproico & 1.500 & 971,600 & 933,100 & 914,100 & 893,900 & 927,600 & $928,06 \pm 28,65$ \\
\hline Amitriptilina & 75 & 33,850 & 32,650 & 33,200 & 34,660 & 37,130 & $34,30 \pm 1,75$ \\
\hline Biperideno & 10 & 6,870 & 3,710 & 3,570 & 3,460 & 3,440 & $4,21 \pm 1,49$ \\
\hline Bupropiona & 300 & - & 246,400 & 235,300 & 280,800 & 311,400 & $268,48 \pm 34,56$ \\
\hline Carbamazepina & 1.000 & 554,800 & 534,000 & 543,100 & 549,200 & 549,800 & $546,18 \pm 7,97$ \\
\hline Carbonato de lítio & 623 & 997,600 & 939,600 & 894,300 & 867,500 & 887,800 & $917,36 \pm 52,02$ * \\
\hline Clomipramina & 100 & 62,470 & 60,590 & 54,800 & 55,090 & 57,000 & $57,99 \pm 3,41$ \\
\hline Clonazepam & 8 & 2,450 & 2,390 & 2,350 & 2,370 & 2,450 & $2,40 \pm 0,05$ \\
\hline Clorpromazina & 300 & 116,300 & 101,600 & 97,760 & 94,370 & 90,850 & $100,18 \pm 9,86$ \\
\hline Diazepam & 10 & 6,620 & 6,120 & 6,160 & 6,240 & 6,430 & $6,31 \pm 0,21$ \\
\hline Fenitoína & 300 & 223,800 & 219,500 & 224,100 & 236,300 & 239,500 & $228,64 \pm 8,72$ \\
\hline Fenobarbital & 100 & 126,500 & 124,000 & 124,400 & 131,600 & 130,200 & $127,34 \pm 3,42$ * \\
\hline Fluoxetina & 20 & 28,2600 & 27,760 & 28,650 & 29,640 & 31,860 & $29,23 \pm 1,62$ * \\
\hline Haloperidol & 8 & 9,640 & 9,260 & 9,120 & 8,790 & 9,580 & $9,28 \pm 0,35$ * \\
\hline Imipramina & 100 & 48,520 & 46,630 & 45,380 & 40,710 & 42,790 & $44,81 \pm 3,09$ \\
\hline Levodopa & 600 & 441,900 & 472,500 & 427,500 & 516,500 & 764,200 & $524,52 \pm 138,3$ \\
\hline Levomepromazina & 300 & 56,370 & 53,100 & 47,330 & 47,740 & 39,730 & $48,85 \pm 6,35$ \\
\hline Naltrexona & 50 & 55,590 & 55,600 & 57,130 & 55,530 & 50,910 & $54,95 \pm 2,36$ * \\
\hline Nitrazepam & 5 & 9,900 & 8,880 & 8,580 & 8,500 & 8,400 & $8,85 \pm 0,61$ * \\
\hline Nortriptilina & 75 & 55,110 & 45,570 & 46,000 & 38,880 & 42,800 & $45,67 \pm 5,99$ \\
\hline Periciazina & 50 & 30,900 & 24,910 & 24,710 & 25,500 & 24,410 & $26,09 \pm 2,72$ \\
\hline Risperidona & 5 & - & 2,950 & 2,610 & 2,790 & 2,690 & $2,76 \pm 0,15$ \\
\hline Sertralina & 50 & 106,000 & 91,410 & 76,740 & 77,090 & 80,630 & $86,37 \pm 12,48$ * \\
\hline Tioridazina & 300 & 149,700 & 116,80 & 117,600 & 109,40 & 102,300 & $119,16 \pm 18,17$ \\
\hline
\end{tabular}

DDD: dose diária definida da Organização Mundial da Saúde; DDP: dose diária prescrita (dose real prescrita para o paciente).

Fonte: dados de consumo coletados do banco de dados online Hygia (http://www.hygia.com.br/).

* DDP > DDD 
Tabela 3

Variação do consumo dos fármacos sujeitos a controle especial entre o ano de 2012 e a média dos anos de 2008-2011, segundo a DDP (dose real prescrita para o paciente).

\begin{tabular}{|c|c|c|c|c|c|c|c|}
\hline \multirow[t]{2}{*}{ Fármaco } & \multicolumn{7}{|c|}{ DDP } \\
\hline & 2008 & 2009 & 2010 & 2011 & Média 2008-2011 & 2012 & Variação \\
\hline Ácido valproico & 971,600 & 933,100 & 914,100 & 893,900 & $928,175 \pm 33,08$ & 927,600 & $-0,062$ \\
\hline Amitriptilina & 33,850 & 32,650 & 33,200 & 34,660 & $33,59 \pm 0,86$ & 37,130 * & 10,54 \\
\hline Biperideno & 6,870 & 3,710 & 3,570 & 3,460 & $4,402 \pm 1,65$ & 3,440 & $-21,86$ \\
\hline Bupropiona & - & 246,400 & 235,300 & 280,800 & $254,167 \pm 23,72$ & 311,400 * & 22,52 \\
\hline Carbamazepina & 554,800 & 534,000 & 543,100 & 549,200 & $545,275 \pm 8,91$ & 549,800 & 0,83 \\
\hline Carbonato de lítio & 997,600 & 939,600 & 894,300 & 867,500 & $924,75 \pm 56,96$ & 887,800 & $-3,99$ \\
\hline Clomipramina & 62,470 & 60,590 & 54,800 & 55,090 & $58,237 \pm 3,88$ & 57,000 & $-2,12$ \\
\hline Clonazepam & 2,450 & 2,390 & 2,350 & 2,370 & $2,39 \pm 0,04$ & $2,450 *$ & 2,51 \\
\hline Clorpromazina & 116,300 & 101,600 & 97,760 & 94,370 & $102,507 \pm 9,66$ & $90,850 * *$ & $-11,37$ \\
\hline Diazepam & 6,620 & 6,120 & 6,160 & 6,240 & $6,285 \pm 0,23$ & 6,430 & 2,31 \\
\hline Fenitoína & 223,800 & 219,500 & 224,100 & 236,300 & $225,925 \pm 7,23$ & $239,500 *$ & 6,01 \\
\hline Fenobarbital & 126,500 & 124,000 & 124,400 & 131,600 & $126,625 \pm 3,49$ & 130,200 * & 2,82 \\
\hline Fluoxetina & 28,2600 & 27,760 & 28,650 & 29,640 & $28,577 \pm 0,80$ & 31,860 * & 11,49 \\
\hline Haloperidol & 9,640 & 9,260 & 9,120 & 8,790 & $9,202 \pm 0,35$ & $9,580 *$ & 4,10 \\
\hline Imipramina & 48,520 & 46,630 & 45,380 & 40,710 & $45,31 \pm 3,33$ & 42,790 & $-5,56$ \\
\hline Levodopa & 441,900 & 472,500 & 427,500 & 516,500 & $464,6 \pm 39,36$ & 764,200 * & 64,49 \\
\hline Levomepromazina & 56,370 & 53,100 & 47,33 & 47,740 & $51,135 \pm 4,37$ & $39,730 * *$ & $-22,30$ \\
\hline Naltrexona & 55,590 & 55,600 & 57,130 & 55,530 & $55,962 \pm 0,78$ & $50,910 * *$ & $-9,03$ \\
\hline Nitrazepam & 9,900 & 8,880 & 8,580 & 8,500 & $8,965 \pm 0,64$ & 8,400 & $-6,30$ \\
\hline Nortriptilina & 55,110 & 45,570 & 46,000 & 38,880 & $46,39 \pm 6,66$ & 42,800 & $-7,74$ \\
\hline Periciazina & 30,900 & 24,910 & 24,710 & 25,500 & $26,505 \pm 2,95$ & 24,410 & $-7,90$ \\
\hline Risperidona & - & 2,950 & 2,610 & 2,790 & $2,783 \pm 0,17$ & 2,690 & $-3,35$ \\
\hline Sertralina & 106,000 & 91,410 & 76,740 & 77,090 & $87,81 \pm 13,92$ & 80,630 & $-8,18$ \\
\hline Tioridazina & 149,700 & 116,80 & 117,600 & 109,40 & $123,375 \pm 17,93$ & $102,300 * *$ & $-17,08$ \\
\hline
\end{tabular}

DDP: dose diária prescrita (dose real prescrita para o paciente).

Fonte: todas os dados referentes ao consumo de medicamentos nos anos indicados foram retirados do banco de dados online Hygia (http://www.hygia.com.br/).

* Valor de DDP 2012 > média DDP 2008-2011;

** Valor de DDP $2012<2008-2011$. 
Tabela 4

Variação do consumo dos fármacos sujeitos a controle especial no período estudado, segundo classificação ATC, DDD/1.000PD, DDD75\%/1.000PD.

\begin{tabular}{|c|c|c|c|c|c|}
\hline Classificação ATC & Fármaco & $\begin{array}{c}\text { Média DDD/1.000PD } \\
2008-2011\end{array}$ & $\begin{array}{c}\text { Variação } \\
\text { DDD/1.000PD } \\
\text { 2012/2008-2011 }\end{array}$ & $\begin{array}{c}\text { Média } \\
\text { DDD75\%/1.000PD } \\
\text { 2008-2011 }\end{array}$ & $\begin{array}{c}\text { Variação } \\
\text { DDD75\%/1.000PD } \\
\text { 2012/2008-2011 }\end{array}$ \\
\hline N03AG01 & Ácido valproico & $1,005 \pm 0,11$ & 22,59 * & $1,340 \pm 0,14$ & 21,12 * \\
\hline N06AA09 & Amitriptilina & $2,798 \pm 0,57$ & $-8,48$ & $3,731 \pm 0,76$ & $-9,57$ \\
\hline N04AA02 & Biperideno & $0,579 \pm 0,08$ & $-3,93$ & $0,772 \pm 0,11$ & $-5,02$ \\
\hline N06AX12 & Bupropiona & $0,011 \pm 0,01$ & 27,27 & $0,015 \pm 0,01$ & 29,55 \\
\hline N03AF01 & Carbamazepina & $1,274 \pm 1,39$ & 92,54 & $1,699 \pm 1,86$ & 90,23 \\
\hline N05AN01 & Carbonato de lítio & $0,642 \pm 0,06$ & 16,26 * & $0,857 \pm 0,09$ & 14,74 * \\
\hline N06AA04 & Clomipramina & $0,354 \pm 0,06$ & 28,63 * & $0,473 \pm 0,08$ & 26,92 * \\
\hline N03AE01 & Clonazepam & $1,693 \pm 0,44$ & 37,06 * & $2,257 \pm 0,58$ & 35,38 * \\
\hline N05AA01 & Clorpromazina & $0,715 \pm 0,01$ & $-0,31$ & $0,953 \pm 0,02$ & $-1,41$ \\
\hline N05BA01 & Diazepam & $8,020 \pm 0,33$ & 1,03 & $10,695 \pm 0,44$ & $-0,14$ \\
\hline N03AB02 & Fenitoína & $1,111 \pm 0,01$ & 6,61 * & $1,482 \pm 0,02$ & 5,33 * \\
\hline N03AA02 & Fenobarbital & $2,913 \pm 0,11$ & $-0,18$ & $3,883 \pm 0,14$ & $-1,37$ \\
\hline N06AB03 & Fluoxetina & $14,93 \pm 1,15$ & $-18,53$ * & $19,897 \pm 1,53$ & $-19,49$ * \\
\hline N05AD01 & Haloperidol & $2,019 \pm 0,04$ & 5,91 * & $2,692 \pm 0,05$ & 4,61 * \\
\hline N06AA02 & Imipramina & $0,819 \pm 0,08$ & 38,63 * & $1,092 \pm 0,11$ & 37,03 * \\
\hline N04BA02 & Levodopa & $0,334 \pm 0,23$ & 87,57 * & $0,299 \pm 0,14$ & 34,67 \\
\hline N05AA02 & Levomepromazina & $0,027 \pm 0,00$ & 8,41 * & $0,032 \pm 0,01$ & 17,83 \\
\hline N07BB04 & Naltrexona & $0,019 \pm 0,01$ & 170,13 * & $0,079 \pm 0,11$ & 167,96 * \\
\hline N05CD02 & Nitrazepam & $0,211 \pm 0,02$ & 12,66 * & $0,267 \pm 0,06$ & 17,23 \\
\hline N06AA10 & Nortriptilina & $0,328 \pm 0,16$ & 128,61 * & $0,454 \pm 0,18$ & 117,84 * \\
\hline N05AC01 & Periciazina & $0,174 \pm 0,01$ & 10,66 * & $0,176 \pm 0,07$ & 43,95 \\
\hline N05AX08 & Risperidona & $0,298 \pm 0,03$ & 129,79 * & $0,397 \pm 0,36$ & 127,14 * \\
\hline N06AB06 & Sertralina & $7,960 \pm 5,00$ & 98,58 * & $10,614 \pm 6,67$ & 92,28 * \\
\hline N05AC02 & Tioridazina & $0,103 \pm 0,00$ & 1,69 & $0,138 \pm 0,00$ & 0,00 \\
\hline
\end{tabular}

ATC: classificação Anatômico Terapêutico e Química.

DDD/1.000PD: dose diária definida/1.000 habitantes/dia; DDD75\%/1.000PD: dose diária definida/1.000 habitantes/dia considerando 75\% da população da cidade.

Fonte: todas os dados referentes ao consumo de medicamentos nos anos indicados foram retirados do banco de dados online Hygia (http://www.hygia.com.br/)

* Variação significativa correspondente ao valor de DDD/1.000PD ou DDD75\%/1.000PD entre o ano de 2012 e média 2008-2011. 
Tabela 5

Comparação entre a taxa de crescimento populacional e o consumo de medicamentos sujeitos à controle especial dispensados no Município de Ribeirão Preto, São Paulo, Brasil, no período de 2008 a 2012.

\begin{tabular}{|c|c|c|c|c|c|}
\hline \multirow[t]{2}{*}{ Variáveis } & \multicolumn{4}{|c|}{ Taxa de crescimento } & \multirow[t]{2}{*}{ Valor de p } \\
\hline & 2009 & 2010 & 2011 & 2012 & \\
\hline Crescimento populacional & 0,00891 & 0,07383 & 0,01266 & 0,0121 & \\
\hline \multicolumn{6}{|l|}{ Fármaco } \\
\hline Ácido valproico & 0,11148 & 0,12613 & 0,27573 & $-0,06145$ & 0,248 \\
\hline Amitriptilina & 0,14143 & 0,03310 & $-0,16052$ & $-0,00102$ & 0,564 \\
\hline Biperideno & $-0,04000$ & 0,05720 & 0,02405 & 0,06980 & 0,564 \\
\hline Bupropiona & - & 4,72414 & $-0,74096$ & 0,69767 & 0,480 \\
\hline Carbamazepina & 0,04416 & 0,02160 & 0,03606 & 0,03996 & 1,000 \\
\hline Carbonato de lírio & 0,20334 & 0,16435 & 0,02584 & 0,09109 & 0,083 \\
\hline Clomipramina & 0,13799 & 0,34281 & 0,18506 & $-0,04516$ & 0,248 \\
\hline Clonazepam & 0,26914 & 0,22157 & 0,21764 & 0,08048 & 0,043 * \\
\hline Clorpromazina & 0,08048 & 0,06653 & 0,02298 & 0,07978 & 0,564 \\
\hline Diazepam & $-0,01088$ & $-0,03295$ & 0,01466 & 0,00291 & 0,083 \\
\hline Fenitoína & $-0,01957$ & 0,04192 & 0,00128 & 0,08036 & 0,386 \\
\hline Fenobarbital & 0,42963 & 0,00731 & $-0,04012$ & 0,02935 & 0,564 \\
\hline Fluoxetina & 0,06331 & $-0,02714$ & $-0,02562$ & $-0,09857$ & 0,083 \\
\hline Haloperidol & 0,00360 & 0,04029 & 0,02991 & $-0,00819$ & 0,248 \\
\hline Imipramina & 0,04366 & 0,01934 & 0,66770 & 0,03916 & 0,564 \\
\hline Levodopa & 0,08995 & 1,79612 & 0,00347 & 0,09343 & 0,248 \\
\hline Levomepromazina & $-0,04049$ & 0,15190 & 0,10256 & 0,27243 & 0,248 \\
\hline Naltrexona & $-0,34884$ & 0,64286 & 0,15217 & 1,81132 & 0,248 \\
\hline Nitrazepam & 0,36029 & 0,00541 & $-0,04839$ & 0,03390 & 0,564 \\
\hline Nortriptilina & 1,55340 & 0,45247 & 1,17976 & $-0,02002$ & 0,248 \\
\hline Periciazina & 0,05275 & 0,12735 & $-0,00370$ & 0,05948 & 1,000 \\
\hline Risperidona & - & 5,67361 & 0,34443 & 0,22678 & 0,034 * \\
\hline Sertralina & 0,67893 & 1,48607 & 0,67305 & 0,12011 & 0,021 * \\
\hline Tioridazina & $-0,04$ & 0,00321 & 0,08626 & $-0,03529$ & 0,146 \\
\hline
\end{tabular}

Nota: taxa de crescimento do fármaco superior à taxa de crescimento populacional. Análise estatística: teste não paramétrico de Mann-Whitney. Fonte: Instituto Brasileiro de Geografia e Estatística 12; Hygia (http://www.hygia.com.br/).

* Valor de $p<0,05$. 


\section{Discussão}

Aproximadamente um em cada cinco pacientes que retiraram medicamentos nas farmácias públicas do Município de Ribeirão Preto usou pelo menos um psicofármaco, sendo a maioria mulheres. SianiMorello et al. 13, ao entrevistarem pacientes de Ribeirão Preto atendidos nas farmácias dos estabelecimentos de atenção primária à saúde, corroboraram os achados do presente trabalho, uma vez que mulheres, idosos e fumantes compreenderam os grupos com a maior probabilidade de usar medicamentos sujeitos a controle especial, notadamente antidepressivos e ansiolíticos. Na mesma linha, o estudo de Quintana et al. 14 evidenciou que a prevalência de uso de medicamentos psicotrópicos entre mulheres foi maior do que entre homens, especialmente em relação aos antidepressivos de segunda geração e ansiolíticos. A menor prevalência de utilização de psicotrópicos no referido estudo quando comparado ao presente $(6,55 \%$ vs. $18,2 \%)$ pode ser explicada possivelmente pela menor proporção de idosos $(16,7 \%$ vs. $29,4 \%) 14$.

Foi observado que fluoxetina, sertralina, diazepam e fenobarbital foram os medicamentos com o maior consumo no município estudado, considerando o consumo total, medido pela DDD/1.000PD e DDD75\%/1.000PD, no ano de 2012. Luis et al. 15 verificaram que mais da metade dos pacientes com doenças mentais atendidos em unidades da Estratégia Saúde da Família (ESF) da região oeste de Ribeirão Preto apresentaram transtornos de humor $(42,2 \%)$ e ansiedade $(16,2 \%)$, dos quais, $77,2 \%$ receberam prescrição de ansiolíticos, anticonvulsivantes ou antiepiléticos $(55,5 \%)$, antidepressivos $(19,2 \%)$ e estabilizador de humor (2,5\%). Esses achados corroboraram o levantamento nacional realizado pela PNAUM, em que os antidepressivos e ansiolíticos estavam entre as classes farmacológicas mais utilizadas na atenção primária no Brasil 2.

Apesar da taxa de consumo de clonazepam ter superado a de crescimento populacional, observouse redução na dose de $1,794 \mathrm{mg} /$ dia com o aumento de um ano na idade e DDP média inferior à DDD da OMS. No entanto, houve aumento de $0,084 \mathrm{mg} /$ dia na dose do diazepam, porém, com DDP média inferior à DDD da OMS. Em 2001, o diazepam foi o psicotrópico mais consumido em Ribeirão Preto $(\mathrm{DDD} / 10.000=96,8) 16$.

Os benzodiazepínicos são prescritos na prática clínica como ansiolíticos, hipnóticos e sedativos, embora o clonazepam também tenha atividade anticonvulsivante. Exceto para a última indicação, são considerados potencialmente impróprios para idosos, uma vez que aumentam o risco de quedas e fraturas 17 , além do uso crônico ter sido identificado como fator de risco para a ocorrência de demência 8 .

Os inibidores seletivos da recaptação de serotonina (ISRS) são alternativas terapêuticas mais seguras para os transtornos de humor e ansiedade. O protocolo clínico preconizado pelo Instituto Nacional para Saúde e Cuidados de Excelência de Reino Unido (NICE - National Institute for Health and Care Excellence) recomenda que os ISRS sejam prescritos como primeira linha de tratamento da depressão em todos os grupos etários 18 . Esse fato pode justificar a menor taxa de crescimento do clonazepam e diazepam quando comparada à sertralina, além da DDP média desta ter sido superior à DDD da OMS.

Embora tenha sido observado aumento da DDP para a fluoxetina no ano de 2012 em comparação à média dos anos de 2008-2011, o que poderia indicar que o tempo de tratamento médio com a fluoxetina reduziu ou a dose média do fármaco cresceu, o consumo total diminuiu (aproximadamente 20\%) no período analisado. Apesar da DDP média ter sido mantida acima da DDD da OMS, é a opção menos segura da classe dos ISRS em função da meia vida longa e por ser um potente inibidor da enzima CYP2D6 19, o que faz com que sua utilização seja desencorajada principalmente em idosos. Os antidepressivos tricíclicos (amitriptilina) também aumentam os riscos de eventos adversos devido à falta de seletividade no bloqueio da recaptação de serotonina. Assim, dentre as opções terapêuticas padronizadas no município, a sertralina é a mais segura para a população idosa, pois inibe moderadamente as enzimas do citocromo P450 e tem meia-vida de eliminação menor, podendo estar menos envolvida com interações medicamentosas e eventos adversos a medicamentos.

A análise dos parâmetros farmacocinéticos deve ser considerada para a indicação de antidepressivos na população idosa, uma vez que, geralmente, compreendem pacientes que apresentam múltiplas comorbidades e que necessitam de polifarmácia. Por conseguinte, o uso concomitante de medicamentos que apresentam interações farmacocinéticas pode interferir na efetividade do tratamento. Esses 
argumentos podem explicar o crescimento significativo de pacientes em uso de sertralina, bem como a redução da dose do clonazepam e do consumo de fluoxetina.

Não obstante, é importante salientar que os antidepressivos, anticonvulsivantes e os antipsicóticos também podem ser utilizados como adjuvantes na terapêutica antinocioceptiva. A clomipramina, fenitoína, amitriptilina, nortriptilina, carbamazepina e ácido valproico contemplam o arsenal terapêutico descrito no protocolo clínico e diretrizes terapêuticas da dor crônica 20.

Como não foi possível analisar o histórico clínico dos pacientes no período estudado, não se pode afirmar para quais condições clínicas esses medicamentos foram dispensados. No entanto, SianniMorello et al. 13 identificaram que, para 35,6\% dos pacientes que retiraram amitriptilina nas farmácias públicas de Ribeirão Preto, a indicação de uso foi para manejo de dor.

Os maiores aumentos dos consumos, tanto em DDD/1.000PD quanto em DDD75\%/1.000PD, no entanto, foram identificados para naltrexona, risperidona, nortripitilina e sertralina. A naltrexona é um medicamento que promove antagonismo em receptores opioides indicados para o tratamento da dependência de opioides e de álcool, e o aumento do consumo poderia indicar a maior abrangência de políticas de saúde pública para o tratamento da adição por estas substâncias 21. Observa-se, no entanto, uma redução da DDP, o que poderia estar associado à flexibilização de regimes de dosagem para aumentar a adesão do paciente.

A risperidona, de acordo com os Protocolos Clínicos e Diretrizes Terapêuticas, é indicada para o tratamento de esquizofrenia refratária 22. Para que a sua dispensação pudesse ser feita nas farmácias do CBAF de Ribeirão Preto para outros transtornos mentais, era necessário que a receita do medicamento viesse acompanhada da "Justificativa do Uso da Risperidona".

As indicações off-label da risperidona incluem tratamento de delirium e de discinesia tardia induzida por neurolépticos. Luis et al. 14 estimaram que 14,2\% da população atendida nas ESF da região oeste de Ribeirão Preto receberam diagnóstico de esquizofrenia e delirium. A despeito do aumento do consumo do antipsicótico atípico, este não superou o uso dos típicos, uma vez que a DDD/1.000PD e DDD75\%/1.000PD da clorpromazina e haloperidol foram superiores à da risperidona no ano de 2012. Além disso, a DDP do haloperidol foi a única superior à DDD da OMS dentre os antipsicóticos padronizados. Esses dados podem indicar que a risperidona dispensada pelo CBAF pode estar sendo prescrita para indicações off-label.

O aumento do consumo dos medicamentos bupropiona, levodopa e risperidona podem ser explicados, por sua vez, pela incorporação na relação municipal do CBAF durante o período analisado. Durante os anos de 2008 e 2009 23,24, a associação disponível de agentes dopaminérgicos era levodopa $250 \mathrm{mg}+$ carbidopa $25 \mathrm{mg}$. Nos anos posteriores, foram padronizadas as associações $25,26,27$ : levodopa $100 \mathrm{mg}$ + benserazida $25 \mathrm{mg}$; levodopa $200 \mathrm{mg}$ + benserazida $50 \mathrm{mg}$; levodopa $200 \mathrm{mg}$ + carbidopa $50 \mathrm{~g}$ e levodopa 50mg + carbidopa 50mg.

Com relação às diferenças nas doses médias usadas por homens e mulheres, estas podem ser explicadas pelas características hormonais de cada sexo. As mulheres apresentam maior propensão biológica e psicossocial a terem mais transtornos de humor e de ansiedade, já os homens são mais susceptíveis à esquizofrenia 28,29. Isso pode explicar por que as mulheres usaram doses maiores de diazepam e os homens clorpromazina.

Por fim, é necessário conhecimento clínico para a correta interpretação da DDP, que pode ser diferente da DDD da OMS. Embora a DDD da OMS seja baseada nas dosagens aprovadas com dados de resultados clínicos de ensaios controlados, a DDP é variável e depende de fatores como gravidade da doença, peso corporal, diferenças interétnicas no metabolismo do medicamento e cultura de prescrição dos profissionais da saúde 30 . Portanto, as discrepâncias identificadas no presente estudo não podem ser interpretadas como subdosagem ou sobredosagem do psicofármaco. Todavia, o acompanhamento do consumo, com o intuito de identificar discrepâncias significativas entre DDP e DDD da OMS, pode ser uma estratégia efetiva para triar pacientes elegíveis ao cuidado farmacêutico. Isso porque os desvios no padrão de uso podem estar associados à morbimortalidade relacionada ao uso de psicofármacos. O acompanhamento farmacoterapêutico contribuiria para o uso seguro e adequado desses produtos. 


\section{Limitações}

O perfil na prescrição de medicamentos sujeitos a controle especial depende de vários fatores, como políticas de saúde, condições sociais e cultura local. Portanto, os dados do presente trabalho devem ser interpretados com cautela para outras realidades, apesar dos dados corroborarem a literatura. Além disso, como foi realizada análise retrospectiva do registro de dispensação de medicamentos, os dados podem estar superestimados, pois a retirada do medicamento não está associada, necessariamente, com a utilização pelo paciente. Além disso, qualquer falta de medicamento que tenha ocorrido durante o período analisado não pôde ser avaliada, pois esta informação não era registrada no sistema. Outra questão importante a ser ressaltada é que como os registros no Hygia são inseridos por diferentes funcionários, isto pode ocasionar falhas na inserção de dados na plataforma, tanto em relação à dispensação dos medicamentos quanto sobre o cadastro do paciente.

Não obstante, esses achados descrevem comportamento de uso semelhante aos obtidos na literatura e podem corroborar a hipótese da medicamentalização excessiva dos transtornos de humor, os quais podem estar relacionados a problemas sociais e econômicos. Desse modo, estimar o consumo de antidepressivos e benzodiazepínicos também pode ser considerado indicador da qualidade da assistência à saúde prestada na atenção básica dos municípios brasileiros, a fim de implementar a prevenção quaternária nestes estabelecimentos.

\section{Conclusão}

Um em cada cinco pacientes que retiraram medicamentos do componente básico de Ribeirão Preto utilizava psicofármacos, sendo a maioria mulheres. Os dados sugerem que, apesar do consumo total de medicamentos sujeitos a controle especial ter aumentado, somente a sertralina, clonazepam e risperidona apresentaram crescimento de consumo superior à taxa de crescimento populacional. As discrepâncias entre DDD da OMS e a DDP são esperadas, porém, quando significativas, podem ser utilizadas como estratégia para a triagem de pacientes elegíveis ao cuidado farmacêutico, com o intuito de contribuir para o uso seguro e apropriado destes produtos. 


\section{Colaboradores}

Todos os autores contribuíram na concepção, projeto, análise e interpretação dos dados; na redação do artigo e revisão crítica relevante do conteúdo intelectual; na aprovação final da versão a ser publicada; e são responsáveis por todos os aspectos do trabalho na garantia da exatidão e integridade de qualquer parte da obra.

\section{Informações adicionais}

ORCID: Júlia Raso Ferreira de Oliveira (00000001-5479-6719); Fabiana Rossi Varallo (00000003-4016-1442); Marcela Jirón (0000-0001-68002246); Iahel Manon de Lima Ferreira (0000-00020468-5399); Manuela Roque Siani-Morello (00000002-5497-2775); Vinícius Detoni Lopes (00000002-9747-9462); Leonardo Régis Leira Pereira (0000-0002-8609-1390).

\section{Referências}

1. Fontana AM. Manual de clínica em psiquiatria. Rio de Janeiro: Editora Atheneu; 2005.

2. Secretaria de Ciência, Tecnologia e Insumos Estratégicos, Ministério da Saúde. Componente avaliação dos serviços de assistência farmacêutica básica: resultados. Brasília: Ministério da Saúde; 2017.

3. Prado MAMB, Francisco PMSB, Barros MBA. Uso de medicamentos psicotrópicos em adultos e idosos residentes em Campinas, São Paulo: um estudo transversal de base populacional. Epidemiol Serv Saúde 2017; 26:111-222.

4. The PLOS Medicine Editors. The paradox of mental health: over-treatment and under-recognition. PLoS Med 2013; 10:e1001456.

5. Linden M, Lecrubier Y, Bellantuono C, Benkert O, Kisely S, Simon G. The prescribing of psychotropic drugs by primary care physicians: an international collaborative study. J Clin Psychopharmacol 1999; 19:132-40.

6. Madruga CS, Paim TL, Palhares HN, Miguel AC, Massaro LTS, Caetano R, et al. Prevalence of and pathways to benzodiazepine use in Brazil: the role of depression, sleep, and sedentary lifestyle. Braz J Psychiatry 2019; 41:44-50.

7. Mendes KCC. O uso prolongado de benzodiazepínicos: uma revisão da literatura [Monografia de Conclusão de Curso]. Belo Horizonte: Universidade Federal de Minas Gerais; 2013.

8. He Q, Chen X, Wu T, Li L, Fei X. Risk of dementia in long-term benzodiazepine users: evidence from a meta-analysis of observational studies. J Clin Neurol 2019; 15:9-19.

9. World Health Organization. Depression and other common mental disorders: global health estimates. Geneva: World Health Organization; 2017.

10. Alduhishy M. The overprescription of antidepressants and its impact on the elderly in Australia. Trends Psychiatry Psychother 2018; 40:241-3.

11. Bezerra IC, Gondim APS, Lima LL, Vasconcelos MGF. "Fui lá no posto e o doutor me mandou foi pra cá": processo de medicamentalização e (des)caminhos para o cuidado em saúde mental na atenção primária. Interface (Botucatu) 2014 ; 18:61-74.

12. Instituto Brasileiro de Geografia e Estatística. Ribeirão Preto: estimativa da população https://cidades.ibge.gov.br/brasil/sp/ribeirao -preto/panorama (acessado em 24/Jun/2019).

13. Siani-Morello MR, Pereira LB, Ferreira IM, Aliste MJ, Pereira LRL. Psychoactive drugs in the Brazilian public health system: use profile and associated factors Braz. J Pharm Sci 2020; [no prelo].

14. Quintana MI, Andreoli SB, Moreira FG, Ribeiro WS, Feijo MM, Bressan RA, et al. Epidemiology of psychotropic drug use in Rio de Janeiro, Brazil: gaps in mental illness treatments. PLoS One 2013; 8:e62270. 
15. Luis MAV, Barbosa SP, Souza J, Vedana KGG, Zanetti ACG, De Azevedo MJ M. Mental health needs and psychoactive drug use in a user population of the family health strategy (FHS) in Ribeirão Preto, São Paulo, Brazil. Community Ment Health J 2017; 54:664-71.

16. Sebastião ECO, Pelá IR. Consumo de psicotrópicos: Análise das prescrições ambulatoriais como base para estudos de problemas relacionados com medicamentos. Pharm Pract 2004; 2:250-66.

17. American Geriatrics Society 2019 updated AGS beers criteria for potentially inappropriate medication use in older adults. J Am Geriatr Soc 2019; 67:674-94.

18. The National Institute for Health and Care Excellence. Depression in adults: recognition and management. https://www.nice.org.uk/guid ance/cg90 (acessado em 24/Jun/2019).

19. Spina E, Santoro V, D’arrigo C. Clinically relevant pharmacokinetic drug interactions with second-generation antidepressants: an update. Clin Ther 2008; 30:1206-27.

20. Ministério da Saúde. Portaria no 1.083, de 2 de outubro de 2012. Aprova o protocolo clínico e diretrizes terapêuticas da dor crônica. Diário Oficial da União 2012; 3 out.

21. Associação Médica Brasileira. Projeto diretrizes: abuso e dependência dos opioides e opiáceos. https://diretrizes.amb.org.br/_Bibliote caAntiga/abuso_e_dependencia_de_opioides. pdf (acessado em 14/Mai/2020).

22. Ministério da Saúde. Portaria no 364, de 9 de abril de 2013. Aprova o Protocolo Clínico e Diretrizes Terapêuticas - esquizofrenia. Diário Oficial da União 2013; 10 abr.
23. Secretaria Municipal de Saúde de Ribeirão Preto. Relação Municipal de Medicamentos Essenciais (REMUNE). Ribeirão Preto: Secretaria Municipal de Saúde; 2008.

24. Secretaria Municipal de Saúde de Ribeirão Preto. Relação Municipal de Medicamentos Essenciais (REMUNE). Ribeirão Preto: Secretaria Municipal de Saúde; 2009.

25. Secretaria Municipal de Saúde de Ribeirão Preto. Relação Municipal de Medicamentos Essenciais (REMUNE). Ribeirão Preto: Secretaria Municipal de Saúde; 2010.

26. Secretaria Municipal de Saúde de de Ribeirão Preto. Relação Municipal de Medicamentos Essenciais (REMUNE). Ribeirão Preto: Secretaria Municipal de Saúde; 2011.

27. Divisão de Farmácia, Secretaria Municipal de Saúde de Ribeirão Preto. Dados relativos às médias de atendimentos nas farmácias da rede pública de saúde do Município de Ribeirão Preto. Ribeirão Preto: Secretaria Municipal de Saúde; 2012.

28. Seeman MV. The role of rstrogen in schizophrenia. J Psychiatry Neurosci 1996; 21:123-7.

29. Gogos A, Sbisa AM, Sun J, Gibbons A, Udawela M, Dean B. A role for estrogen in schizophrenia: clinical and preclinical findings. Int J Endocrinol 2015; 2015:615356.

30. World Health Organization; WHO International Working Group for Drug Statistics Methodology; WHO Collaborating Centre for Drug Statistics Methodology; WHO Collaborating Centre for Drug Utilization Research and Clinical Pharmacological Services. Introduction to drug utilization research. Geneva: World Health Organization; 2003. 


\section{Abstract}

The consumption of psychotropic drugs is considered a public health problem, due to the potential for addiction and the occurrence of adverse events. In this context, the current study aimed to characterize the consumption of psychotropic medications dispensed in primary healthcare units in Ribeirão Preto, São Paulo State, Brazil. This ecological study consulted the Hygia database from 2008 to 2012. The following variables were extracted: psychotropic drugs dispensed, amount dispensed per year, and patients'sex and age bracket. For each psychotropic drug, we calculated the defined daily dose per 1,000 inhabitants/day (DDD/1,000PD), defined daily dose per 1,000 inhabitants/day considering $75 \%$ of the population (DDD75\%/1,000PD) who withdrew medicines through the Brazilian Unified National Health System (SUS), and the prescribed daily dose (PDD). The study compared the population growth rate to the growth in the medicines' consumption. A total of 1,577,241 patients were identified who withdrew medications during the study period, of whom 287,373 (18.2\%) used at least one drug subject to special control. There was an increase in the total consumption of psychotropic drugs (DDD/1,000PD), but comparison to the population growth rate showed that only sertraline $(p=0.021)$, risperidone $(p=0.034)$, and clonazepam $(p=0.043)$ presented higher growth rates. The PDD for seven drugs were higher than the World Health Organization (WHO) DDD. Identifying discrepancies between DDD and PDD can be useful as a strategy for screening patients eligible for pharmaceutical care, since they can contribute to the prevention of morbidity and mortality related to medications.

Psychotropic Drugs; Pharmacoepidemiology; Drug Utilization; Unified Health System

\section{Resumen}

El consumo de psicofármacos está considerado un problema de salud pública, debido al potencial de dependencia y ocurrencia de eventos adversos. En este contexto, el presente estudio tuvo como objetivo caracterizar el consumo de psicofármacos dispensados en unidades básicas de salud de Ribeirão Preto, São Paulo, Brasil. Se realizó un estudio ecológico, con consulta a la base de datos Hygia de 2008 a 2012. Se extrajeron las variables: psicofármaco dispensado, cantidad dispensada al año, sexo y franja de edad de los pacientes. Para cada psicofármaco se calculó la dosis diaria definida por 1.000 habitantes/día (DDD/1.000PD), la dosis diaria definida por 1.000 habitantes/ día, considerándose un $75 \%$ de la población (DDD75\%/1.000PD) que consiguieron el medicamento por el Sistema Único de Salud (SUS) y la dosis diaria prescrita (DDP). Se comparó la tasa de crecimiento poblacional con la de crecimiento por consumo de medicamentos. Se identificaron a 1.577.241 pacientes que obtuvieron medicamentos durante el periodo evaluado, entre los cuales $287.373(18,2 \%)$ utilizaron por lo menos uno sujeto a un control especial. Hubo un aumento en el consumo total de los psicofármacos (DDD/1.000PD), no obstante, tras la comparación con la tasa de crecimiento poblacional, solamente la de consumo de sertralina $(p=0,021)$, risperidona $(p=0,034) y$ del clonazepam $(p=0,043)$ fueron superiores. Las $D D P$ de siete fármacos eran mayores a la DDD de la Organización Mundial de la Salud. Las discrepancias entre DDD y DDP pueden ser útiles como estrategia para triar pacientes elegibles para el cuidado farmacéutico, puesto que pueden contribuir a la prevención de morbimortalidad relacionada con el uso de medicamentos.

Psicotrópicos; Farmacoepidemiologia; Utilización de Medicamentos; Sistema Único de Salud
Recebido 30/Mar/2020

Versão final reapresentada em 04/Jun/2020

Aprovado em 12/Jun/2020 